\title{
New adaptation method based on cross layer and TCP over protocols to improve QoS in mobile ad hoc network
}

\author{
Moulay Hicham Hanin, Mohamed Amnai, Youssef Fakhri \\ Laboratory of Computer Science, Ibn Tofail University, Morocco
}

\begin{tabular}{l} 
Article Info \\
\hline Article history: \\
Received Mar 21, 2020 \\
Revised Dec 11, 2020 \\
Accepted Dec 21, 2020 \\
\hline
\end{tabular}

\section{Keywords:}

Ad-hoc

AODV

Congestion

Cross-layer

DSDV

OLSR

TCP

\begin{abstract}
Due to rapid growth of multimedia traffic used over the mobile ad-hoc networks (MANETs), to keep up with the progress of this constraint MANETs protocols becoming increasingly concerned with the quality of service. In view of the random mobility nodes in MANET, TCP becomes more unreliability in case of higher energy consumption and packet loss. In this paper we proposed a new optimization approach to enhance decision making of TCP based on some changes of IEEE 802.11 MAC uses cross layer approach. The aim is to minimize the impact of retransmissions of packet lost and energy consumption in order to analyse and chose the appropriate routing protocol for TCP that can be enhance QoS MANET. Our simulation results based QoS study using NS3 show that, our proposal achieved better performance of TCP in MANETs significantly, and also improved the throughput, energy consumption and facilitates the traffic transmission over routing protocol.
\end{abstract}

This is an open access article under the CC BY-SA license.

\section{Corresponding Author:}

Hanin Moulay Hicham

Laboratory of Computer Science

Ibn Tofail University Kenitra, Morocco

Email: moulay.hicham.hanin@uit.ac.ma

\section{INTRODUCTION}

In recent years the technology of MANET become in the spotlight for researchers due to the flexibility of implementation. More to these advantages, MANET can be connected to internet without any prior configuration and self-repairing data communication mobile devices. Likewise, MANET could be integrated to draw a connection between reach infrastructure fixed or cellular. This last, the latter led to challenges to ensure best routing of packets. Moreover, this symbolizes a difficulty notice in the case of incorporate a better result between the guarantee of performance and reliability.

First of all, MANETs is widely used in military and disaster areas and can be used in various field, and applications used in mobile computing (nomadic computing applications). The mobile ad hoc networks have exceptional lineaments which make it more suitable to implement for multiple intend. These features are disseminating mechanism, autonomous architecture, reconfigurable network topology and light weight terminals. So, there are various issues occurring during designing and implementing of mobile-ad hoc network (MANET) such as security, reliability, and power consumption. Therefore, QoS provisioning for MANET becomes very challenging task. Whereas the amount of multimedia traffic on the Internet is increasing, a significant large portion is still generated by transmission control protocol (TCP) based applications such as file transfer protocol (FTP) and HTTP. Therefore, efficient support of TCP is crucial for these types of networks. Hence, TCP has been found not to perform well in MANETs as usually reported in literature [1]. 
Indeed, TCP works reliably in wired networks and the majority of packets lost justify by network congestion [2]. Indeed, the packets are dropped when does not have enough space in waiting queues or almost full. Consequently, the packet losses can be considered as an indicator of congestion in wired networks. However, in MANET where losses usually happen reasons, such as a result of nodes are not stable, distrust link quality or interference. Nevertheless, is not able to differentiate between reasons of packet losses due to specific features of MANET and losses due to congestion.

The foremost problem confronts by TCP in MANETs as regards packet loss and retransmits a lost packet that is not generated by the network. In wired networks the error rates are low compared to other networks, thereabouts all versions of TCP currently guess that degrade the quality of radio links are owing to attenuation of signal and interference. So, TCP decelerate the sending rate by regulating its congestion window, when a packet is detected to be lost, owing to timeout or triple duplicate ACKs. Nevertheless, various problems like as capture effect, frequent link changes, spatial reuse, hidden or exposed node, contention with TCP ACK packets and routing protocol, all participate to the evil performance of TCP MANETs. To enhance TCP performance in MANETs, some solutions are proposed like as cross layer [3], layered [4] and other transport protocols [5] have been suggested for MANET.

The paper is organized as follows. In section 2, we investigate and describe the existing survey papers and the methods that were used as base to realize our contribution. In Section 3, we introduce our proposed. Simulation results are included in Section 4 to evaluate and confirm process of our contribution by dint to simulations and results obtained. In Section 5, concludes the paper and discusses some estimate to extensions of our work.

\section{RELATED WORK}

A lot of studies research has conduct investigation behaviour versions of TCP in both wired and wireless communication (mobile or static) networks to ameliorate the quality of service in TCP's functioning [6-10]. But most ancient propositions are designed for wireless cellular networks or wired networks $[11,12]$ here the only link join the mobile node [13-15] and the base station. However, however, MANETs are multi-hop networks in which data have to be diffused in different wireless links, between the sender and receiver. Latterly, new proposition have been discussed [16-18] at the level of MANET, the majority generate quality results but did not optimize accordingly, new study have been proposed to enhance and give more adaptive results.

Feng et al. [19] proposed I-AODV protocol to select the next hop at the level of the routing protocol algorithm, they focused on the state of the node and considered as a best selection means. Demonstrate that the minimum hops not consistently condition to select the optimal path. Another method to improve QoS in MANET routing proposed by Hanin et al. [20] based on fuzzy logic to reduce the number of redundant retransmissions while diffusing a broadcast message in the network. In the same objective, Hamzaoui et al. [21] proposed a new algorithm used clustering and k-means methods to guarantee high levels of QoS when the network becomes dense, larger and dynamic.

Kanellopoulos [22] allowed the lower layer MANET to detect and make a difference between possible network events. He proposed an overview of Congestion control based on Cross-layer optimization that can improves the overall network performance. Mahadevan [23] proposed three principal points such as threshold-based data transmission, queuing modeling, and channel modeling over physical layer to support quality of service, and provides the information about channel layer path loss but is not justify between reasons of packet losses.

Prathviraj et al. [24] proposed routing algorithm to reduce load on the route establishment based on multilayer of TCP/IP in order to enhance QoS in MANET, but they did not take into consideration the divergence of routing protocols in MANET. Kliazovich and Granelli [25] is focused on the diversification of the path in the routing with a quality of service, he proposed the dynamic source routing (DSR) with a decision stratified, which did not take into consideration the case of a dense network

\section{PROPOSED METHOD}

The focus of this work, we present a new enhancement technique for improving QoS in MANET. This approach includes two main components which are as follows:

- The solution of cross layer depend on the energy, signal strength and noise, In this section, we present a new modification based on the idea of the transversal layer and inheritance of the IEEE 802.11 MAC standard with TCP new reno by taking into account the different modes of mobility for performing effective packet loss control. 
- Interaction between routing protocols and new hybrid TCP in MANET analyse the performance of routing protocol in terms of packet loss ratio, throughput and energy consumption and taken into account new hybrid TCP.

\subsection{The signal strength}

Mobility and connection between nodes in MANET networks is the greatest of all defiances face TCP MANETs. Caused by mobility the link is frequently broken due to results in packet lack or failed. TCP in its current version packet congestion will often justify packet losses at the TCP protocol level. Indeed, replaying the packets until they successfully arrive at the receiver is not sufficient to validate this assumption, one hand, and on the other, it lifts the delay, which is why the assumption is erroneous even though it minimizes the transmission window, the network performance is degraded. Klemm et al. [26] suggest modalities that are dependent on strength to succeed these packet losses owing to mobility idea revolves around the following principles:

- If link breakdown based on the measures signal strength then probably due to a remoteness from neighbouring, response to this situation, it make possible the provisional use of the transmission power to maintain and re-establish the link.

- Establish a path rediscovery proactively before break down the path, if the signal strength measurements indicate that the interrelation is probability to lack.

\subsection{RTT method}

The majority traffic transmitted on the internet rely on TCP to delivery data over the internet. Principal goal of TCP is to make sure that reliable communication over an inaccurate network layer and to provide orderly data from sender to receiver precisely. Until now, a lot of research works have been done on TCP and various version of TCP have been deployed [27, 28]. Various routing mechanism have great impact on the QoS of MANET as it was mentioned. Every TCP variant has different mechanism for congestion control and loss recovery. TCP-RENO is one of the fruitful attempts it can not only enhances the forwarding process, nevertheless the mechanism based on congestion avoidance. When a source reduces its speed the losses necessary occur, by reason of the fact that congestion of TCP processing instruction is growing the congestion window with time and decrease it just when there are losses. The key idea of TCP-Reno is that: as illustrated in the Figure 1, the round-trip time (RTT) can be assessed by source for a connection using (1).

$$
\operatorname{RTT}(\mathrm{n}+1)=\beta \operatorname{RTT}(n)+(1-\beta) M
$$

- $\mathrm{M}$ : The inevitable time of a segment dismissal the ACK

- $\alpha$ : The stable value between 0 and 1 , control how fast the suitable RTT changes.

- RTT (n): The value of RTT issued by last packet dispatched.

- RTT (n+1): depending on this estimated value of RTT (n 1) and the experimental value of RTT, TCP RENO will compared them and take their differences as a variable named DIFF.

We extended the methodology used in [29] in order to the measure the TCP computational energy consumption. By TCP we first started to calculate the total energy and then we underground the energy due to the transmission and reception of data on the node and the inactive energy

\subsection{Proposed solution: hybrid TCP with interaction routing protocols}

As previously indicated and in accordance with [30], the TCP is not really consistent with mobile ad hoc networks, because that TCP envisage all packets loss as suffer deterioration the quality of network and congestion. Consequently, many approaches have been proposed to solve this problem of compliance [31]. The majority of these proposals are based on cross layer solutions which run away from the pure between principal model OSI of communications with virtually strict limit among layers. In order to have a dynamic compensation method through layer boundaries, several cross layer approach was proposed. The aim of our paper is to offer a cross layer solution based on the RTT and Benefit the force of the wireless signal to enhance the behaviour of TCP in MANETs. The idiosyncrasy of the proposal is that it takes into consideration the outside particularities of the network with the impact of routing protocols.

In our approach, we proposed the factor of the signal strength to identify distance between nodes in MANET. This factor it used to calculate the estimated value of RTT and compare it with RTT in the regular case so as to identify the reason of the loss and take action under analysis. 

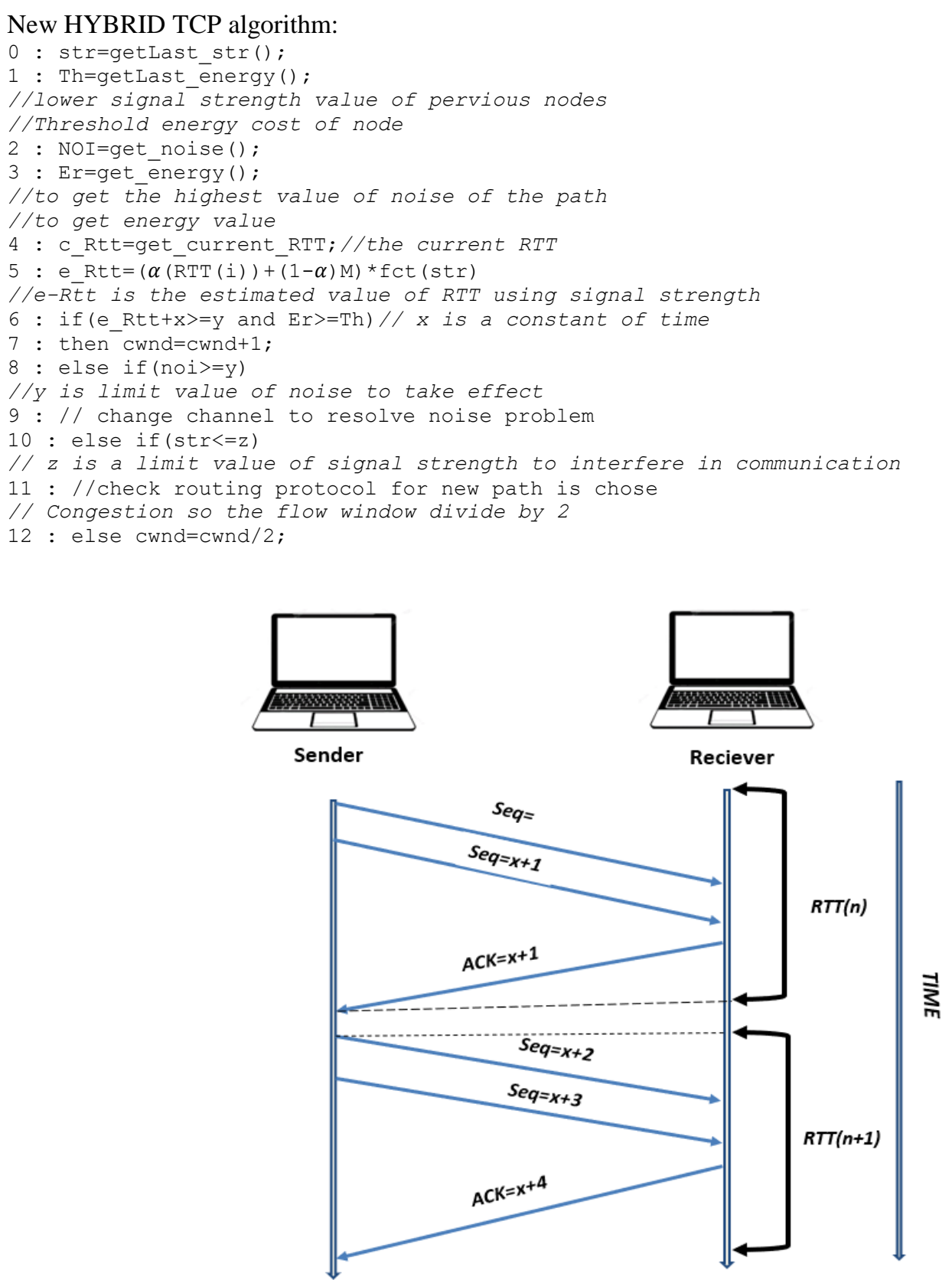

Figure 1. RTT evaluation scenario and TCP

\section{RESULTS AND DISCUSSION}

Regarding the simulation, the NS3 simulator was used due to its large library of TCP versions in order to represent several scenarios in MANET. The TCP simulation scenarios are implemented with the parameters cited in the Table 1. In our simulation, as shown in Figure 2 we create a network congestion based on five nodes, and the packet loss define in node that in middle. This occurs by Data traffic flows required to go through intermediate node in order to get receiver. One should also note that, different levels of data congestion can be generated by controlling the number of TCP data flows traversing this given network node at a precise moment. We pick MANET topology with number of nodes between 10 and 50 means of the mobile routing OLSR and AODV protocol. The signal strength value is varying.

In second step we measure the packet loss rate of normal_TCP and new_hybrid_TCP over OLSR, AODV and DSDV. In the Figures 3-6, the results demonstrate that the OLSR use new_hybrid_TCP gives a good feedback compared to normal_TCP. And OLSR protocol perform better than AODV and DSDV. These results is due to the fact that new hybrid TCP takes in consideration the energy, effect of signal strength changes, the difference between it and a congestion, which makes the network work properly. 
Table 1. Parameter values

\begin{tabular}{cccc}
\hline Parameter & Value & Parameter & Value \\
\hline Channel type & Wireless Channel & Receiving Power & $1.4 \mathrm{w}$ \\
MAC & 802.11 & Idle Power & $1.1 . \mathrm{W}$ \\
Routing Protocol & OLSR, DSDV, AODV & Sleep Power & $0.002 \mathrm{w}$ \\
Speed & $10,20,30,40,50 \mathrm{~m} / \mathrm{s}$ & TC-INTERVAL & $5 \mathrm{sec}$ \\
Transmission Range & $250 \mathrm{~m}$ & Hello-INTERVAL & $3 \mathrm{sec}$ \\
Initial Energy & $100 \mathrm{Joule}$ & Topography & $1000 \mathrm{~m} \times 1000 \mathrm{~m}$ \\
Transmission Power & $1.65 \mathrm{w}$ & Time of simulation & $300 \mathrm{sec}$ \\
\hline
\end{tabular}

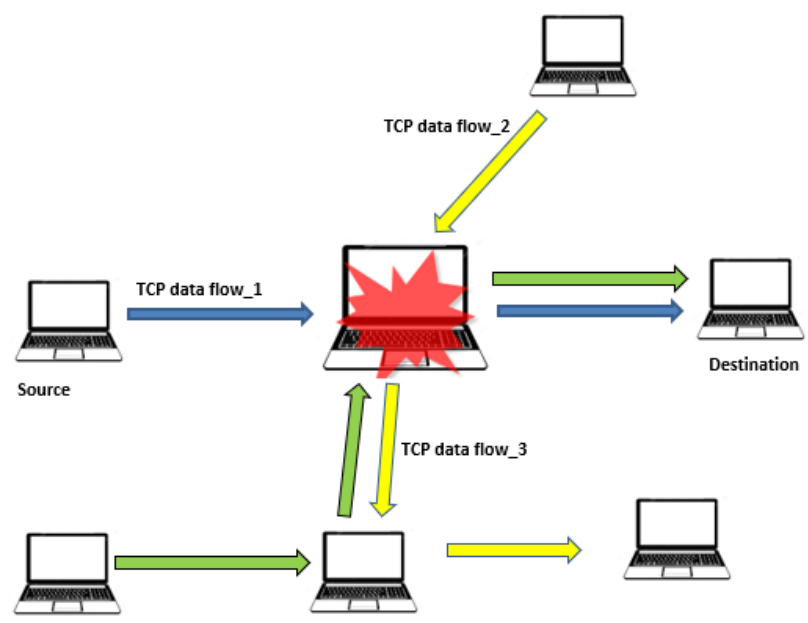

Figure 2. Scenario described the network interference

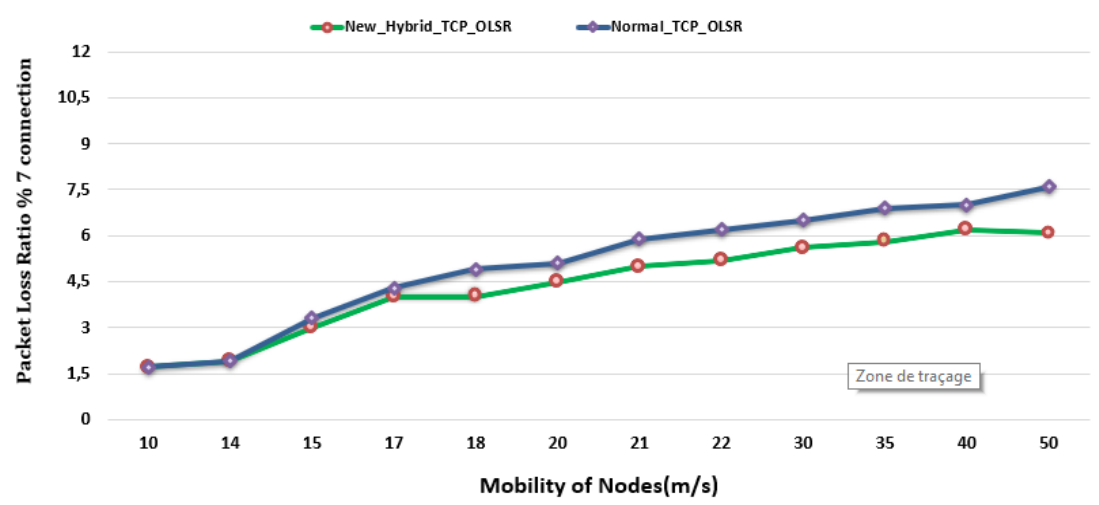

Figure 3. Packet loss rate of new hybrid TCP over OLS

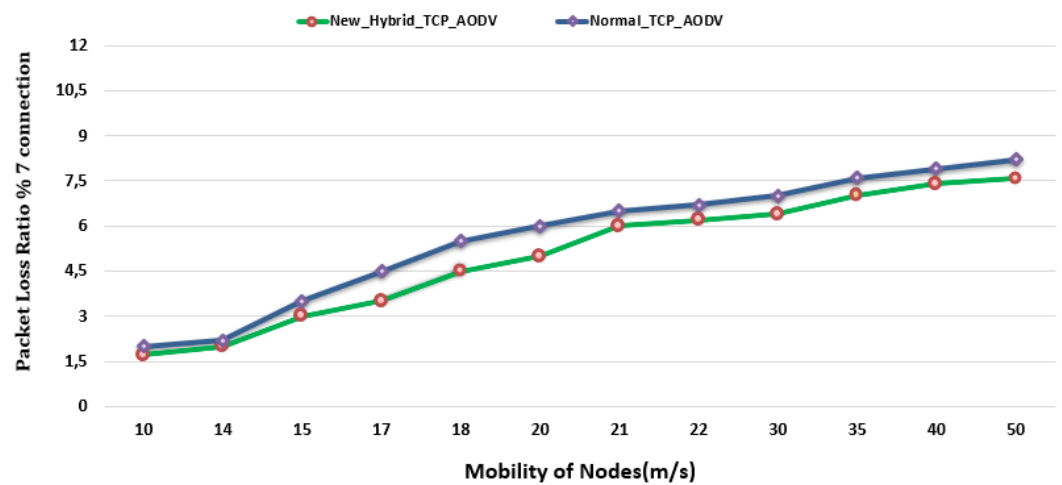

Figure 4. Packet loss rate of new hybrid TCP over AODV 


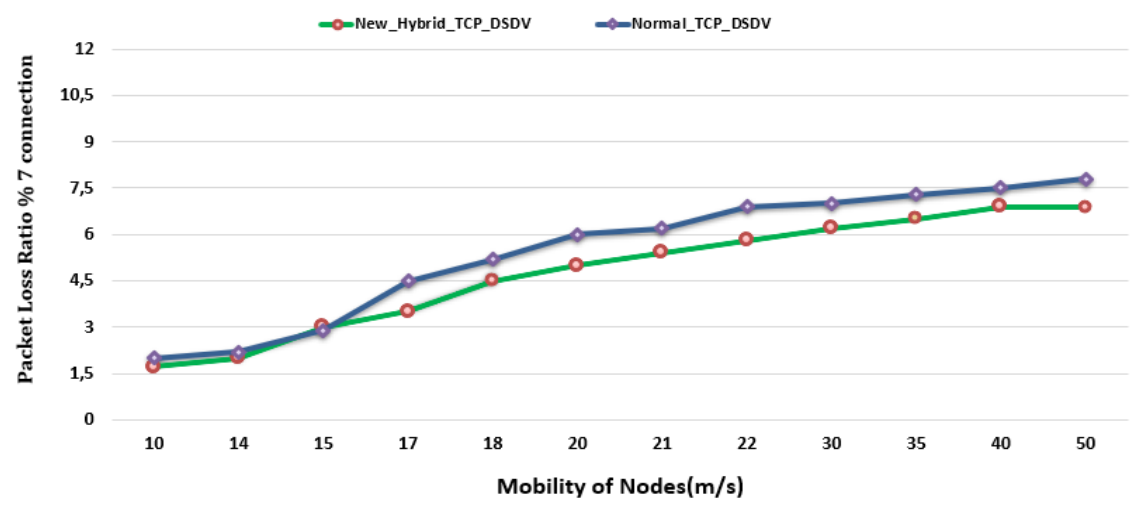

Figure 5. Packet loss rate of new hybrid TCP and normal TCP over DSDV

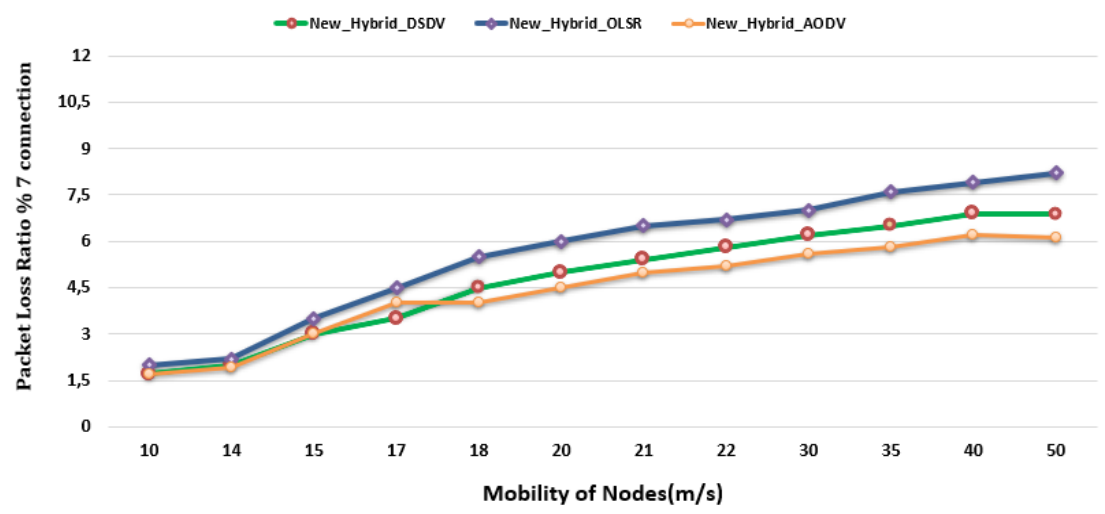

Figure 6. The comparison between OLSR, AODV and DSDV in case of packet loss

Figure 7 show that the OLSR is more suitable in view of calculate throughput (kbps). if we notice Figure 8, can note that the computational energy cost is elevated in case of OLSR protocol compared to case of DSDV and AODV protocol. For the sake of clarity, the Figure 9 illustrate that the new hybrid TCP over DSDV protocol gives a good feedback compared to normal TCP. This result is due to the fact that New Hybrid TCP takes in consideration the nodes mobility, instead of normal TCP the mobility as congestive which degrade throughput. However, as shown in Figure 10 when we use a dynamic ad hoc network with route change we note in case of OLSR consume less than AODV and DSDV.

We use a static ad hoc network without route changes to separate the effect of network traffic interference from the other packet loss reasons. As shown in Figures 11 and 12 the protocol OLSR and AODV consumed more energy than AODV over new TCP hybrid. Caused by routing overhead to route maintenance.

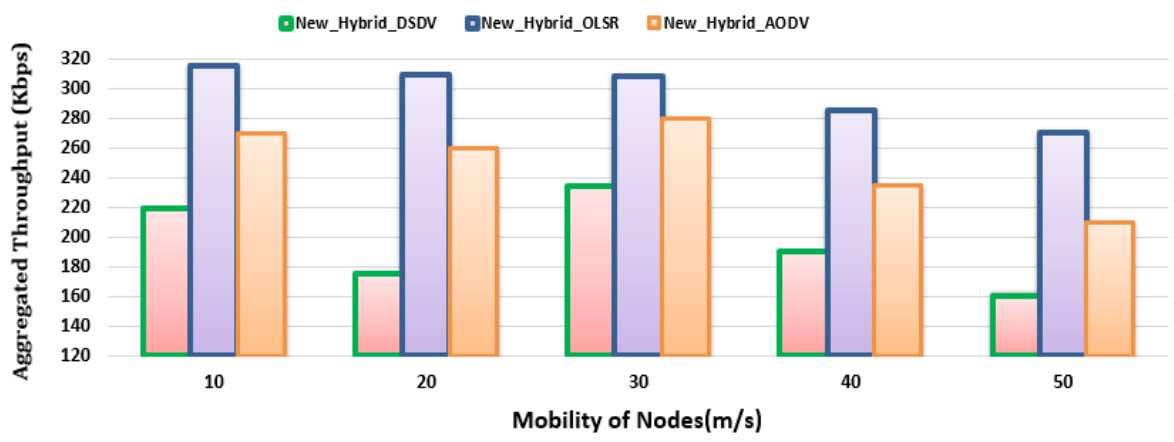

Figure 7. Throughput measured over OLSR, AODV and DSDV with mobility node 


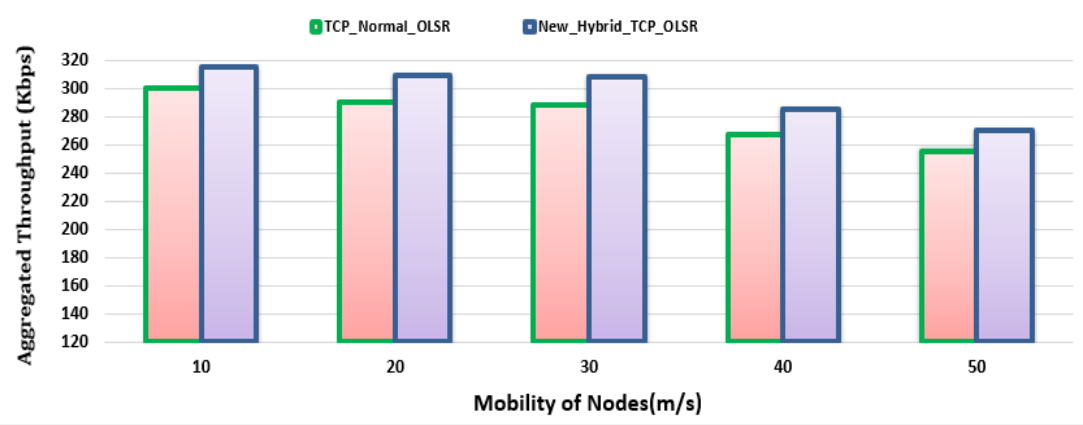

Figure 8. Throughput measured of new hybrid TCP and normal TCP over OLSR

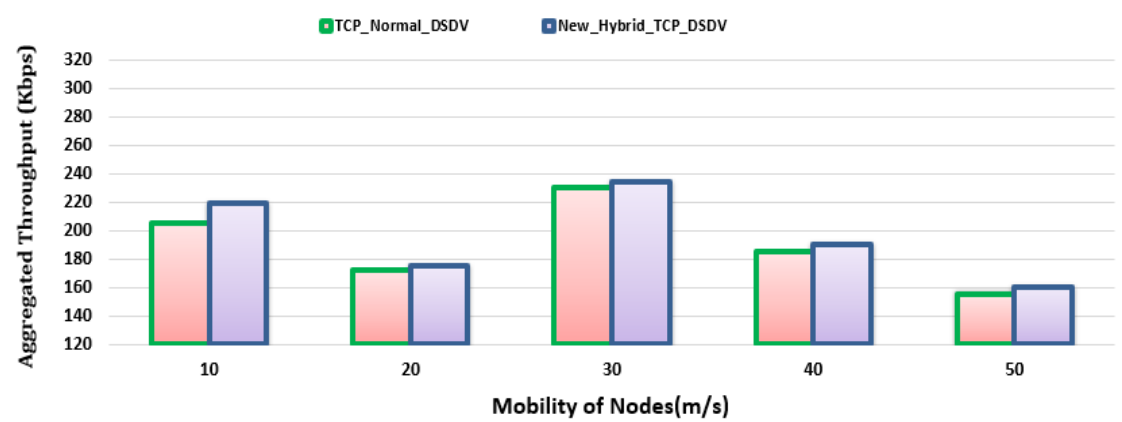

Figure 9. Throughput measured of new hybrid TCP and normal TCP over DSDV

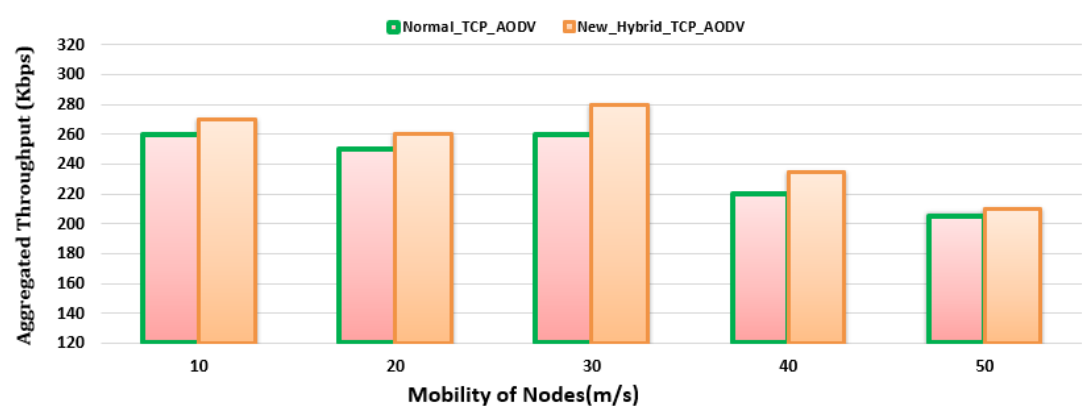

Figure 10. Throughput measured of new hybrid TCP and normal TCP over AODV

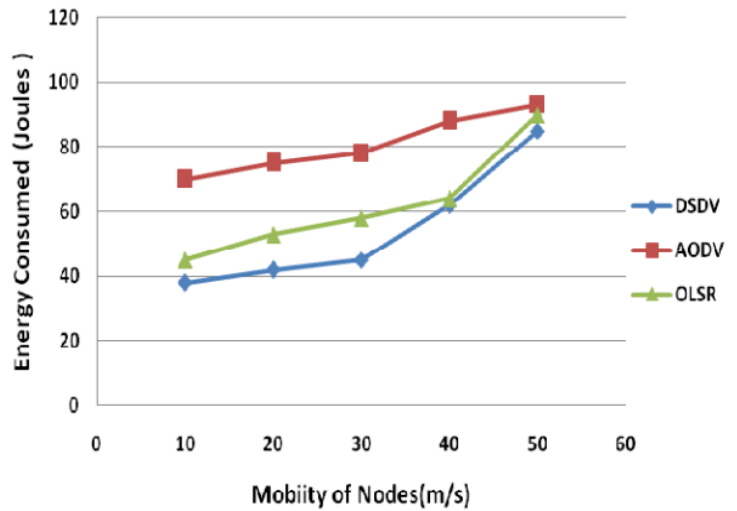

Figure 11. New hybrid TCP over routing protocols OLSR, DSDV and AODV at different node mobility

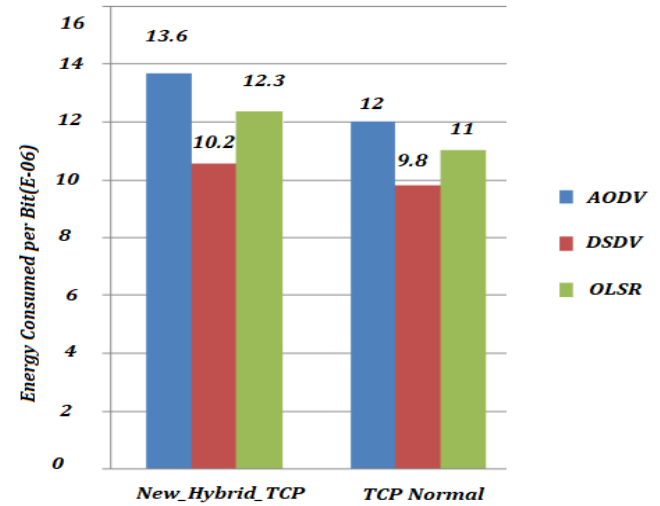

Figure 12. New hybrid TCP over routing protocols OLSR, DSDV and AODV at different node mobility 


\section{CONCLUSION}

In this paper, we have handled the issue of QoS in MANET, includes two main components. We first exposed the inconvenience effects has on TCP performance means to improve it, these leads are to justify between reason of packet losses based on energy, RTT and signal strength over the routing protocols. The second is to select the appropriate routing protocol to this end. Simulation purposed over NS3 demonstrates that OLSR is the best routing protocol given to the packet loss ratio. Otherwise, DSDV consume less energy compared to other protocols. AODV is undesirable protocol attached to energy consumption. In our coming works we will attempt to improve TCP and routing protocol to make it able to support constraint of internet of thing.

\section{REFERENCES}

[1] K. Laeeq, N. Islam, J. Bhayo, "Performance Analysis of TCP and UDP over Mobile Ad hoc Network," Journal of Independent Studies and Research - Computing, vol. 14, no. 1, pp. 12-15, Jan-Jun. 2016.

[2] Lim, Chang-hyeon, and Ju-wook Jang, "An adaptive end-to-end loss differentiation scheme for tcp over wired/wireless networks," International Journal of Computer Science and Network Security (IJCSNS), vol. 7, no. 3, pp. 72-83, 2007.

[3] G. Holland and N. Vaidya, "Analysis of TCP performance over mobile ad hoc networks," ACM Wireless Networks, vol. 8, no. 2, pp. 275-288, 2002

[4] M. Bechler, S. Jaap, and L.C. Wolf, "An Optimized TCP for internet access of vehicular Ad Hoc networks," Proceedings of the 4th International IFIP Networking Conference, 2005.

[5] M. Thottan and M.C. Weigle, "Impact of 802.11e EDCA on mixed TCPbased applications," Proceedings of the International Wireless Internet Conference (WICON), 2006.

[6] A.M. Al-Jubari, M. Othman, B.M. Ali, N.A. Wati and A. Hamid, "TCP performance in multi-hop wireless ad hoc networks: challenges and solution," EURASIP Journal on Wireless Communications and Networking, 2011.

[7] A. Saad, A. Rahem, A. Hussein, A. Mohammed, "Vehicular Ad Hoc Networks: Growth and Survey for Three Layers," International Journal of Electrical and Computer Engineering (IJECE), vol. 7, no. 1, pp. 271-284, 2017, doi: 10.11591/ijece.v7i1.pp271-284.

[8] S. Anbu Karuppusamy and K. Batri, "Improving the performance of TCP in Ad hoc networks based on signal strength and buffering system," Journal of Applied Sciences Research, vol. 8, no. 5, pp. 2554-2563, 2012.

[9] A. Sachan and A. Rajput, "Comparison of TCP Performance on WLAN," Technical report, 2010.

[10] C. Mbarushimana, A. Shahrabi, T. Buggy, "A cross-layer TCP enhancement in QoS-aware mobile ad hoc networks," Journal Computer Networks, vol. 57, no. 1, pp. 286-301, 2013.

[11] G. Marfia, C. Palazzi, G. Pau, M. Gerla, M.Y. Sanadidi and M. Roccetti, "TCP Libra: Exploring RTT-Fairness for TCP," International Conference on Research in Networking, vol. 4479, pp. 1005-1013, 2007.

[12] H. Jiang and C. Dovrolis, "Passive Estimation of TCP Round Trip Times," Newsletter ACM SIGCOMM Computer Communication Review, vol. 32, no. 3, pp. 75-88, 2002, doi: 10.1145/571697.571725.

[13] M. Gunes and D. Vlahovic, "The Performance of the TCP/RCWE Enhancement for Ad-Hoc Networks," Proceedings Seventh International Symposium on Computers and Communications (ISCC"02), 2002, doi: 10.1109/ISCC.2002.1021656.

[14] S. Lohier, Y.G. Doudane and G. Pujolle, "Cross-layer loss differentiation algorithms to improve TCP performance in WLANs," Telecommunication Systems, vol. 36, no. 1-3, pp. 61-72, 2007.

[15] D. Panda, and R. Dash, "Reliability Evaluation and Analysis of Mobile Ad Hoc Networks," International Journal of Electrical and Computer Engineering (IJECE), vol. 7, no. 1, pp. 479-485, 2017, doi: 10.11591/ijece.v7i1.pp479485.

[16] T. Mahmoodi, V. Friderikos, O. Holland and A.H. Aghvami, "CrossLayer Design to Improve Wireless TCP Performance with Link-Layer Adaptation," Proceedings of the 65th IEEE Vehicular Technology Conference (VTC Spring 2007), 2007, pp. 1504-1508, doi: 10.1109/VETECF.2007.320.

[17] Bhagyashri R. Hanji, and Rajashree Shettar, "Cross Layer Solution for Energy and Delay Optimization in MANETs," International Journal of Electrical and Computer Engineering (IJECE), vol. 8, no. 6, pp. 4745-4754, 2018, doi: 10.11591/ijece.v8i6.pp4745-4754.

[18] Feng, D., and Zhu, Y., "An Improved AODV Routing Protocol Based on Remaining Power and Fame," International conference on Electronic Computer Technology, 2009, pp. 117-121, doi: 10.1109/ICECT.2009.117.

[19] H. Chang, H. Kan, M. Ho, "Adaptive TCP congestion control and routing schemes using cross-layer information for mobile ad hoc networks," Computer Communications, vol. 35, no. 4, pp. 454-474, 2012.

[20] MH. Hanin, Y. Fakhri and M. Amnai, "A new efficient technique to enhance quality of service in OLSR protocol," Lecture Notes Advanced Intelligent Systems for Computing Sciences, vol. 5, pp. 85-97, 2019.

[21] Y. Hamzaoui, et al., "Enhancenig OLSR Routing Protocol Using K-Medoids Clustering Method in MANETs," International Journal of Applied Engineering Research (IJAER), vol. 12, no. 2, pp. 200-206, 2017.

[22] D. Kanellopoulos., "Congestion Control for MANETs: An overview," ICT Express, vol. 5, no. 2, pp. 77-83, 2019, doi: 10.1016/j.icte.2018.06.001.

[23] G. Mahadevan, "A combined scheme of video packet transmission to improve cross layer to support QoS for MANET,” Alexandria Eng. J, vol. 57, no. 3, pp. 1501-1508, 2017, doi: 10.1016/j.aej.2017.03.033. 
[24] Prathviraj N., Santosh L., Deshpande, "Rough set based QoS enabled multipath source routing in MANET," International Journal of Electrical and Computer Engineering (IJECE), vol. 10, no. 2, pp. 1915-1923, 2020, doi: 10.11591/ijece.v10i2.pp1915-1923.

[25] D. Kliazovich and F. Granelli, "Cross-layer congestion control in ad hoc wireless networks," Ad Hoc Networks Journal (Elsevier), vol. 4, no. 6, pp. 669-792, 2006, doi: 10.1016/j.adhoc.2005.08.001

[26] F. Klemm, Zhenqiang-Ye, S.V. Krishnamurthy and S.K. Tripathi, "Improving TCP Performance in Ad Hoc Networks using Signal Strength based Link Management," Journal Ad Hoc Networks, vol. 3 no. 2, pp. 175-191, Mar. 2005.

[27] Jacobson, V. "Congestion avoidance and control. Computer Communication," Review, vol. 18, no. 4, pp. 314-329, 1988.

[28] Jacobson, V., "Modifed TCP Congestion Avoidance Algorithm," Technical report, 1990.

[29] B. Wang and S. Singh, "Computational energy cost of TCP," Proceedings of IEEE INFOCOM'04, 2004, doi: 10.1109/INFCOM.2004.1356967.

[30] Mbarushimana, S. and Shahrabi, A., "Comparative study of reactive and proactive routing protocols performance in mobile ad hoc networks," 21st International Conference on Advanced Information Networking and Applications Workshops (AINAW 2007), 2007, pp. 679-684, doi: 10.1109/AINAW.2007.123.

[31] Bhagyashri R. Hanji and Rajashree Shettar, "Cross Layer Solution for Energy and Delay Optimization in MANETs," International Journal of Electrical \& Computer Engineering (IJECE), vol. 8, no. 6, pp. 4745-4754, Dec. 2018.

\section{BIOGRAPHIES OF AUTHORS}

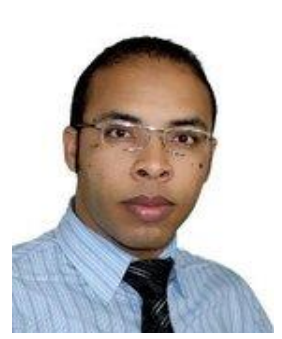

Hanin Moulay Hicham obtained his (DEUG) degree on mathematics in 2006 from Ibn Zohr University, Agadir city, morocco. Then, he received his B.S degree on mathematics and computer science. In 2009, the author got his M.S degree on systems and networks from Ibn Zohr, Agadir, Morocco. The author has been an administrative of systems and networks (2009-2012). Since 2012, he is currently a $\mathrm{PhD}$ researcher of optimization of the service quality in AD-HOC networks. Also, currently doing his research in the Laboratory in Computer Science and Telecommunications (LARIT) Kenitra, Morocco.

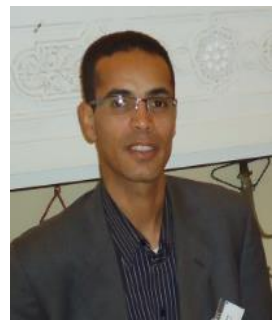

Amnai Mohamed received his bachelor's degree in 2000, in IEEA (Computers, Electronics, Electrical and Automation) from Molay Ismail's University, Errachidia city. Then, the author obtained his master's degree in 2007, from Ibn Tofail University, Kenitra city. In 2011, he received his $\mathrm{PhD}$ on Telecommunication and computer science, from Ibn Tofail University in Kenitra city, Morocco. Since March 2014 Professor, he has been an Assistant at National School of Applied Sciences Khouribga, Settat University, Morocco. He joined the Faculty of Sciences of Kénitra, Department of Computer Science and Mathematics, Ibn Tofail University, Morocco, as an Associate Professor in 2018. The author is also an associate member of Research Laboratory in Computer Science and Telecommunications (LARIT), Team Networks and Telecommunications Faculty of Science, Kenitra, Morocco. He is also an associate member of laboratory IPOSI National School of Applied Sciences, Hassan 1 University, Khouribga, Morocco.

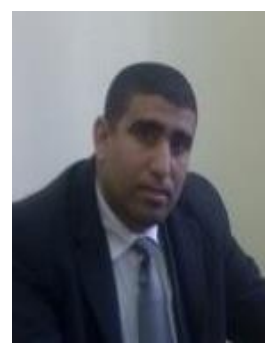

Fakhri Youssef received his Bachelor's Degree (B.S) in Electronic Physics in 2001 and his Master's Degree (DESA) in Computer and Telecommunication from the Faculty of Sciences, University Mohammed V, Rabat, Morocco, in 2003 where he developed his Master's Project at the ICI Company, Morocco. He received a PhD in 2007 from the University Mohammed V Agdal, Rabat, Morocco in collaboration with the Polytechnic University of Catalonia (UPC), Spain. He joined the Faculty of Sciences of Kénitra, Department of Computer Science and Mathematics, Ibn Tofail University, Morocco, as an Associate Professor in Mars 2009, he is the Laboratory head at LARIT, Associate Researcher at the Laboratory for Research in Computing and Telecommunications (LRIT) in the Faculty of Sciences of Rabat, and Member of Pole of Competences STIC Morocco. 